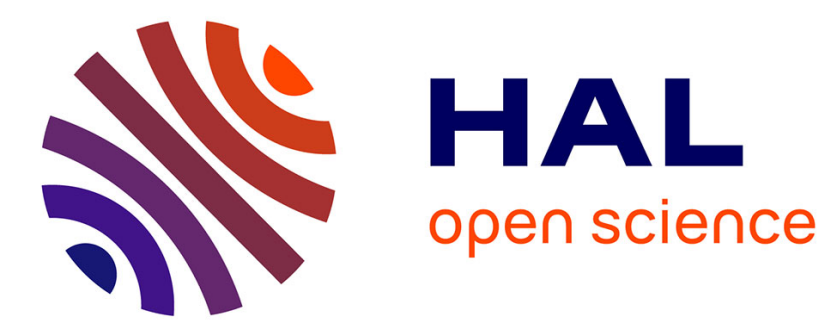

\title{
Passivity based control of irreversible port Hamiltonian Systems.
}

\author{
Héctor Ramirez Estay, Yann Le Gorrec, Bernhard Maschke
}

\section{To cite this version:}

Héctor Ramirez Estay, Yann Le Gorrec, Bernhard Maschke. Passivity based control of irreversible port Hamiltonian Systems.. IFAC Workshop on Thermodynamic Foundations of Mathematical Systems Theory, TFMST'13., Jan 2013, France. pp.1-6. hal-00872215

\section{HAL Id: hal-00872215 https://hal.science/hal-00872215}

Submitted on 11 Oct 2013

HAL is a multi-disciplinary open access archive for the deposit and dissemination of scientific research documents, whether they are published or not. The documents may come from teaching and research institutions in France or abroad, or from public or private research centers.
L'archive ouverte pluridisciplinaire HAL, est destinée au dépôt et à la diffusion de documents scientifiques de niveau recherche, publiés ou non, émanant des établissements d'enseignement et de recherche français ou étrangers, des laboratoires publics ou privés. 


\title{
Passivity Based Control of Irreversible Port Hamiltonian Systems
}

\author{
Hector Ramirez* Yann Le Gorrec* Bernhard Maschke** \\ Françoise Couenne ${ }^{* *}$

\begin{abstract}
* Department of Automation and Micro-Mechatronic Systems, F-25030 Besançon, France. \{ramirez, legorrec\}@femto-st.fr Faculté Sciences et Technologie, France ; CNRS, UMR500\%, Laboratoire d'Automatique et Génie des Procédés, Villeurbanne, F-69622, France (e-mail:\{ramirez,maschke\}@lagep.univ-lyon1.fr)
\end{abstract} \\ FEMTO-ST UMR CNRS 6174, ENSMM, 26 chemin de l'épitaphe, \\ ** Université de Lyon, Lyon, F-69003, France ; Université Lyon 1,
}

\begin{abstract}
The frameworks of thermodynamic availability function and irreversible port Hamiltonian systems are used to derive passivity based control strategies for irreversible thermodynamic systems. An energy based availability function is defined using as generating function the internal energy. This is a variation with respect to previous works where the total entropy usually corresponds to the generating function. The specific structure of irreversible port-Hamiltonian systems then permits to elegantly derive stability conditions for open and closed thermodynamic systems. The results are illustrated on two classical thermodynamic examples: The heat exchanger and the continuous stirred tank reactor.
\end{abstract}

Keywords: Irreversible port Hamiltonian systems, Passivity based control, Irreversible thermodynamics, Entropy creation, CSTR.

\section{INTRODUCTION}

Establishing a relation between irreversible Thermodynamics and system theory is an open and ongoing line of research (Eberard et al., 2007; Favache et al., 2010; Hoang et al., 2011; Ramirez et al., 2013), where one fundamental problem is to find a geometric structure which can represent thermodynamic systems in a similar manner as the symplectic or Poisson structure do for mechanical systems (Brockett, 1977; van der Schaft, 1986; Aris, 1989; Maschke et al., 1992). For stability analysis and control purposes the use of the thermodynamic properties of irreversible systems have been particularly successful when using the concept of available storage (Willems, 1972) which has been specialized to irreversible systems as the availability function (Ydstie and Alonso, 1997; Alonso and Ydstie, 2001; Ydstie, 2002; Hoang et al., 2011, 2012). It has been shown that in the case of homogeneous systems the thermodynamic availability function can be used for stability analysis and in some cases for control purpose. Many extensions of these works to the stability analysis of distributed parameter systems or system networks have also been proposed (Alonso and Ydstie, 1996, 2001; Ydstie, 2002; Jillson and Ydstie, 2007).

Port Hamiltonian systems (PHS) (Maschke and van der Schaft, 1992) have been widely used in modelling and passivity-based control (PBC) of mechanical and electro-

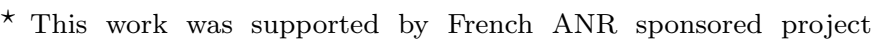
HAMECMOPSYS under Reference Code ANR-11-BS03-0002 and French-Chilean ECOS-CONICYT sponsored project under Reference Code C12E08
}

mechanical systems (Duindam et al., 2009). For those systems, the Hamiltonian function represents the total energy of the system and the energy flows between the different energy domains of the system are represented by a structure matrix. This structure matrix actually defines a Poisson bracket which corresponds to the existence of conservation laws or balance equations for open systems. For instance the conservation of the energy is the base of the control using passivity based control (PBC) methods (Ortega et al., 2002). For physical systems with irreversible phenomena, as for instance chemical reactions or smart materials, which present transformations that involves irreversible entropy creation, it is not sufficient to express only the conservation of energy (first principle of Thermodynamics); it is also necessary to express the irreversible entropy creation (second principle of Thermodynamics) as a system theoretic property. Recently the use of contact geometry and irreversible PHS (IPHS) (Eberard et al., 2007; Favache et al., 2010; Ramirez et al., 2013) have allowed to endow open thermodynamic systems with a geometric and quasi geometric structure respectively with promising perspective for control design.

In this work we combine the results of stability analysis and control design using the availability function with the quasi PHS formulation given by IPHS. To this we derive an energy based availability function defined using the internal energy as generating function, which is a variation from previous works where the total entropy is commonly used as generating function (Alonso and Ydstie, 2001; Ydstie, 2002; Hoang et al., 2011, 2012). The IPHS 
structure then permits to elegantly derive the stability conditions for closed and open thermodynamic systems.

This paper is organized as follows, Section 2 presents the framework of IPHS and Section 3 the energy based availability function and its specialization to IPHS. In Section 4 the approach is illustrated on two classical thermodynamic models: The heat exchanger and the continuous stirred tank reactor (CSTR). Finally Section 5 presents some closing remarks and lines of future work.

\section{IRREVERSIBLE PORT-HAMILTONIAN SYSTEMS}

On the state space $\mathbb{R}^{n} \ni x$, a PHS is defined in the case of power preserving systems by the following state equation,

$$
\dot{x}=J(x) \frac{\partial U}{\partial x}(x)+g(x) u(t)
$$

where $U: \mathbb{R}^{n} \rightarrow \mathbb{R}$ is the Hamiltonian function that is usually the total energy of the system, $J(x) \in \mathbb{R}^{n} \times$ $\mathbb{R}^{n}$ is a skew-symmetric structure matrix, $g(x) \in \mathbb{R}^{m} \times$ $\mathbb{R}^{n}$ is the input vector field and $u(t) \in \mathbb{R}^{m}$ is a time dependent input. For those systems, the Hamiltonian function represents the total electro-mechanical energy of the system and the skew-symmetric structure matrix represents the energy flows between the different energy domains of the system. Furthermore the structure matrix $J(x)$ relates to symplectic geometry as it defines a Poisson bracket, if it satisfies the Jacobi identities, else it is a pseudo-Poisson bracket (see van der Schaft and Maschke (1994)). If $J$ is constant in some local coordinates then it satisfies the Jacobi identities (van der Schaft, 2000). In the sequel we will consider only true Poisson brackets (not pseudo-Poisson brackets). The Poisson bracket of two $C^{\infty}\left(\mathbb{R}^{n}\right)$ functions $Z$ and $G$ is expressed as:

$$
\{Z, G\}_{J}=\frac{\partial Z^{\top}}{\partial x}(x) J(x) \frac{\partial G}{\partial x}(x) .
$$

The PHS dynamics is expressed in term of the Poisson bracket (2) as:

$$
\dot{x}=\{x, U\}_{J}+g(x) u(t) .
$$

The properties of Poisson brackets such as their skewsymmetry and the fact they satisfy Jacobi identities correspond to the existence of conservation laws or balance equations for open systems. For instance the conservation of the energy is the base of the control using PBC methods (Ortega et al., 2002). Several attempts have been made in order to preserve as much as possible the $\mathrm{PH}$ structure, leading to a class of system called quasi PHS Hangos et al. (2001); Otero-Muras et al. (2008); Ramirez et al. (2009); Dörfler et al. (2009); Hoang et al. (2011). These systems retain as much as possible the port Hamiltonian structure, but differ by their structure matrices and input vector fields which depend explicitly on the gradient of the Hamiltonian. An important remark is that, although the forms of PHS (1) and quasi PHS are very similar and both embed, by skew-symmetry of the structure matrix, the conservation of energy, in the latter the drift dynamic is a nonlinear function in the gradient $\frac{\partial U}{\partial x}(x)$. In this sense the symplectic structure of the PHS, given by the Poisson tensor associated with the structure matrix $J(x)$, is destroyed.

There is a large class of thermodynamic systems that can be expressed as quasi PHS if the Hamiltonian function is selected as a thermodynamic potential such as the internal energy or the entropy (Eberard et al., 2007; Favache et al., 2010; Ramirez et al., 2013). From a control perspective it is usually more complicated to impose a desired closed-loop dynamic on quasi PHS. Passivity based techniques can be easily applied to PHS, however due to the non-linearity with respect to the gradient of the Hamiltonian, this is not the case for quasi PHS.

In recent works (Ramirez, 2012; Ramirez et al., 2012, 2013) a class of quasi PHS, denoted Irrevesible Port Hamiltonian Systems (IPHS) have been proposed to model a large class of thermodynamic systems and to embed the first and second principle of Thermodynamics in the structure of the quasi PHS.

Definition 1. (Ramirez, 2012; Ramirez et al., 2013) IPHS are defined by the dynamical equation

$$
\dot{x}=R\left(x, \frac{\partial U}{\partial x}, \frac{\partial S}{\partial x}\right) J \frac{\partial U}{\partial x}(x)+g\left(x, \frac{\partial U}{\partial x}, u\right),
$$

where

(1) $x \in \mathbb{R}^{n}$ is the state vector, $U(x): \mathcal{C}^{\infty}\left(\mathbb{R}^{n}\right) \rightarrow \mathbb{R}$ and $S(x): \mathcal{C}^{\infty}\left(\mathbb{R}^{n}\right) \rightarrow \mathbb{R}$

(2) The structure matrix $J \in \mathbb{R}^{n} \times \mathbb{R}^{n}$ is a constant skewsymmetric matrix.

(3) $R=R\left(x, \frac{\partial U}{\partial x}, \frac{\partial S}{\partial x}\right)$ is composed of a positive definite function and a Poisson bracket evaluated on $S$ and $U$ :

$$
R\left(x, \frac{\partial U}{\partial x}, \frac{\partial S}{\partial x}\right)=\gamma\left(x, \frac{\partial U}{\partial x}\right)\{S, U\}_{J},
$$

with $\gamma\left(x, \frac{\partial U}{\partial x}\right)=\hat{\gamma}(x): \mathcal{C}^{\infty}\left(\mathbb{R}^{n}\right) \rightarrow \mathbb{R}, \hat{\gamma} \geq 0$, a nonlinear positive function of the states and co-states of the system that can be expressed as a function of the states only.

(4) $g\left(x, \frac{\partial U}{\partial x}, u\right) \in \mathbb{R}^{1} \times \mathbb{R}^{n}$ is associated with the port of the system, where the input is $u(t) \in \mathbb{R}^{m}$ a time dependent function.

The main difference with the definition of a PHS is that $R\left(x, \frac{\partial U}{\partial x}, \frac{\partial S}{\partial x}\right)$ depends on the co-state variables destroying the linearity of any Poisson tensor, considering the mapping $\frac{\partial U}{\partial x}$ to the drift dynamics $R\left(x, \frac{\partial U}{\partial x}, \frac{\partial S}{\partial x}\right) J \frac{\partial U}{\partial x}$ and associated with the matrix $R J$. Furthermore, the vector field $g\left(x, \frac{\partial U}{\partial x}, u\right)$ may also depend on states and co-states. The first and second principle express, respectively, the conservation of energy and the irreversible transformation of entropy. It is possible to represent this by the following equations

$$
\frac{d U}{d t}=0 \quad \text { and } \quad \frac{d \mathfrak{S}}{d t}=\sigma\left(x, \frac{\partial U}{\partial x}\right) \geq 0
$$

where the Hamiltonian $U$ is the internal energy, $\mathfrak{S}$ denotes an entropy like function (that may be equal to the total entropy $S$ ) and $\sigma\left(x, \frac{\partial U}{\partial x}\right)$ the irreversible entropy creation which in general depends on the state and the gradient of the total energy. For IPHS, by skew-symmetry of $J$ the total energy of the system satisfies the energy balance equation

$$
\frac{d U}{d t}=\frac{\partial U^{\top}}{\partial x} g(u(t))
$$

Indeed, since $g(u(t))$ represent the flows through the controlled-ports of the system the only energy variation is due to the interaction with the environment. The entropy variation on other hand is given by 


$$
\frac{d S}{d t}=\frac{\partial S}{\partial x}^{\top} R\left(x, \frac{\partial U}{\partial x}, \frac{\partial S}{\partial x}\right) J(x) \frac{\partial U}{\partial x}+{\frac{\partial S^{\top}}{\partial x}}^{\top} g(u(t)) .
$$

A consequence of the second principle of Thermodynamics is that the entropy variation due to internal transformations is always greater or equal to zero. This actually requires $R J$ to explicitly depend on $\frac{\partial U}{\partial x}$,

$$
\frac{\partial S}{\partial x}^{\top} R\left(x, \frac{\partial U}{\partial x}, \frac{\partial S}{\partial x}\right) J(x) \frac{\partial U}{\partial x}=\sigma_{\text {int }} \geq 0,
$$

since this should hold for any generating function $U(x)$.

\section{AVAILABILITY FUNCTION AND EQUILIBRIUM}

In this section the concept of available energy (Willems, 1972 ) is used to propose a class of PBC for IPHS. To this end a variation of the availability function (Ydstie and Alonso, 1997; Alonso and Ydstie, 2001; Ydstie, 2002) is proposed. It is important to remark that, unlike previous works where in general the total entropy is used as generating function (Alonso and Ydstie, 1996, 2001; Ydstie, 2002; Jillson and Ydstie, 2007; Hoang et al., 2011, 2012), we use the internal energy to define an energy based availability function. The reason to use the internal energy is that it corresponds to the Hamiltonian of the IPHS, and hence becomes the natural candidate for the construction of control Laypunov functions. Let us recall some general properties of thermodynamic systems. More details may be found in Callen (1985) or Sandler (2006), among many other textbooks. The variation of the internal energy of a homogeneous system is defined by Gibbs' equation

$$
d U=T d S-P d V+\sum_{i=1}^{m} \mu_{i} d n_{i}
$$

where the extensive variables are the internal energy $U$, the entropy $S$, the volume $V$ and the mole numbers $n_{i}$, and the intensive variables are the temperature $T$, the pressure $P$ and the chemical potentials $\mu_{i}$. By defining the vectors of extensive and intensive variables, $z=\left[S, V, n_{1}, \ldots, n_{m}\right]^{\top}$ and $w=\left[T,-P, \mu_{1}, \ldots, \mu_{m}\right],(8)$ may be written as

$$
d U=w^{\top} d z .
$$

The internal energy $U$ is a homogeneous function of degree 1, so from Euler's Theorem we obtain,

$$
U=w^{\top} z
$$

which implies that $w(z)$ is a homogeneous function of degree 0 of $z$ and $w(z)=\frac{\partial U}{\partial z}$. For homogeneous systems, as a consequence of the second law of Thermodynamics, the internal energy corresponds to a convex function (Callen, 1985; Alonso and Ydstie, 2001). Furthermore the internal energy function is strictly convex with respect to $z$ and independent of the dynamic behaviour of the system as soon as one element of $z$ is fixed (Jillson and Ydstie, 2007).

We emphasise that the internal energy function (and hence also the total entropy function) does not posses a strict minimum. Hence the need of constructing a Lyapunov function with a strict minimum at the desired equilibrium. Identifying $x=z$, where $x$ is the state vector of the IPHS of Definition 1 we define the energy based availability function as

$$
A\left(x, x^{*}\right)=U(x)-\left[U\left(x^{*}\right)+\frac{\partial U}{\partial x}\left(x^{*}\right)^{\top}\left(x-x^{*}\right)\right] \geq 0
$$

where $x^{*}$ is a reference and possibly a desired equilibrium.
Remark 2. Let's note that the only possible value for which $A=0$ is $x=x^{*}$ as soon as one of the extensive variables is fixed (Jillson and Ydstie, 2007).

The time derivative of $A$ is given by

$$
\frac{d A}{d t}=\left(\frac{\partial U}{\partial x}(x)-\frac{\partial U}{\partial x}\left(x^{*}\right)\right)^{\top} \frac{d x}{d t} .
$$

Since the availability function qualifies as Lyapunov function for thermodynamic systems, we may specialize (12) to IPHS as follows:

$$
\begin{aligned}
\frac{d A}{d t}=-\frac{\partial U^{\top}}{\partial x}\left(x^{*}\right) R J & \frac{\partial U}{\partial x}(x)+ \\
& \left(\frac{\partial U}{\partial x}(x)-\frac{\partial U}{\partial x}\left(x^{*}\right)\right)^{\top} g(u) .
\end{aligned}
$$

Now, for closed-systems, i.e., without matter exchange with the environment, the previous equation reduces to

$$
\frac{d A}{d t}=-R \frac{\partial U^{\top}}{\partial x}\left(x^{*}\right) J \frac{\partial U}{\partial x}(x),
$$

from which the following proposition follows.

Proposition 1. The equilibrium point $x^{*}$ is asymptotically stable if:

$$
\left(\frac{\partial U}{\partial x}\left(x^{*}\right)^{\top} J \frac{\partial U}{\partial x}(x)\right)\{S, U\}_{J} \geq 0 .
$$

with strict equality only at $x^{*}$.

Proof. From (14) we obtain by using the definition of $R$

$$
\begin{aligned}
\frac{d A}{d t} & =-R\left(\frac{\partial U}{\partial x}\left(x^{*}\right)^{\top} J \frac{\partial U}{\partial x}(x)\right), \\
& =-\gamma\left(\frac{\partial U}{\partial x}\left(x^{*}\right)^{\top} J \frac{\partial U}{\partial x}(x)\right)\{S, U\}_{J},
\end{aligned}
$$

By positivity of $\gamma$ and since the availability $A$ qualifies as a Lyapunov function for the system it is stable if (15) holds. If $x^{*}$ is an isolated minimum, then asymptotic stability follows invoking La Salle's invariance principle on a region around $x^{*}$.

In the case of controlled thermodynamic systems, Proposition 1 may be extended to controlled IPHS to shift the closed-loop dynamic equilibrium. This is performed similarly as in passivity based control of mechanical systems (Ortega et al., 1998), being the difference that in the later, the natural Lyapunov function of the system is the total energy and in the present case the energy is the generating function of the Lyapunov function.

Proposition 2. The closed-loop equilibrium $x^{*}$ of a controlled IPHS is asymptotically stable if it satisfies:

$$
\begin{aligned}
\gamma\left(\frac{\partial U}{\partial x}\left(x^{*}\right)^{\top} J \frac{\partial U}{\partial x}(x)\right)\{S, U\}_{J} \\
-\left(\frac{\partial U}{\partial x}(x)-\frac{\partial U}{\partial x}\left(x^{*}\right)\right)^{\top} g u \geq 0
\end{aligned}
$$

with strict equality only at $x^{*}, u^{*}$, where $u^{*}$ is the steady state value of the control-input at the desired equilibrium.

Proof. The proof follows directly from Proposition 1 taking into consideration the term $\left(\frac{\partial U}{\partial x}(x)-\frac{\partial U}{\partial x}\left(x^{*}\right)\right)^{\top} g(u)$.

Remark 3. Proposition 2 can be directly used for control design. Indeed it is sufficient to design a control law $u$ 
satisfying (20) such that the closed loop system is stable and converges asymptotically to $x^{*}$.

Proposition 2 may be interpreted as an adaptation of the well known PBC of mechanical systems (Ortega et al., 1998) for the thermodynamic case. In the following section we illustrate on two classical thermodynamic benchmark examples the application of Propositions 1 and 2. It is interesting to notice that the results follow very naturally due to the thermodynamic structure of the system model and Lyapunov function.

\section{EXAMPLES}

In this section we illustrate the results on the model of a heat exchanger and the continuous stirred tank reactor (CSTR). The IPHS deduction of these two systems have been given in details in Ramirez (2012); Ramirez et al. $(2012,2013)$. The main assumption in booth examples is that the mass or volume is constant. This constraint guarantees the strict convexity of the availability function and hence allows to use it as a Lyapunov function.

\subsection{Example: the heat exchanger}

IPHS model (Ramirez et al., 2013) Consider two simple thermodynamic systems, indexed by 1 and 2 (for instance two ideal gases), which may interact only through a conducting wall. The dynamic of this system is given by the following equation

$$
\left[\begin{array}{l}
\dot{S}_{1} \\
\dot{S}_{2}
\end{array}\right]=\lambda\left[\begin{array}{l}
\frac{T_{2}\left(S_{2}\right)}{T_{1}\left(S_{1}\right)}-1 \\
\frac{T_{1}\left(S_{1}\right)}{T_{2}\left(S_{2}\right)}-1
\end{array}\right]+\lambda_{e}\left[\begin{array}{c}
0 \\
\frac{T_{e}(t)}{T_{2}\left(S_{2}\right)}-1
\end{array}\right]
$$

where $S_{1}$ and $S_{2}$ are the entropies of subsystem 1 and 2, $T_{e}(t)$ a time dependent external heat source and $\lambda>0$ and $\lambda_{e}>0$ denotes Fourier's heat conduction coefficients. The temperatures are modelled as exponential functions of the entropies $T\left(S_{i}\right)=T_{0} \exp \left(\frac{S_{i}}{c_{i}}\right)$, where $T_{0}$ and $c_{i}$ are constants. This system may be written as:

$$
\left[\begin{array}{c}
\dot{x}_{1} \\
\dot{x}_{2}
\end{array}\right]=\underbrace{\lambda\left(\frac{1}{\frac{\partial U}{\partial x_{2}}}-\frac{1}{\frac{\partial U}{\partial x_{1}}}\right)}_{R} \underbrace{\left[\begin{array}{cc}
0 & -1 \\
1 & 0
\end{array}\right]}_{J}\left[\begin{array}{l}
\frac{\partial U}{\partial x_{1}} \\
\frac{\partial U}{\partial x_{2}}
\end{array}\right]+\underbrace{\lambda_{e}\left[\begin{array}{c}
0 \\
\frac{1}{\frac{\partial U}{\partial x_{2}}}-\frac{1}{u}
\end{array}\right]}_{g},
$$

where $x=\left[S_{1}, S_{2}\right], U\left(x_{1}, x_{2}\right)=U_{1}\left(x_{1}\right)+U_{2}\left(x_{2}\right)$ is the internal energy of the overall system composed of the addition of the internal energies of each subsystem, $u(t)$ the controlled input that corresponds to the external heat source $T_{e}(t)$. Remark that $\frac{\partial U}{\partial x_{i}}=T_{i}\left(x_{i}\right)$. This system has been widely studied from a modelling perspective using the contact Hamiltonian framework in (Eberard et al., 2007). It is possible to write the model of the heat exchanger as a IPHS (4) as follows

$$
\dot{x}=R(x, T) J T(x)+g(T, u(t)) u(t),
$$

where $T(x)=\left[T_{1}\left(x_{1}\right), T_{2}\left(x_{2}\right)\right], R(x, T(x))=\lambda\left(\frac{1}{T_{2}}-\frac{1}{T_{1}}\right)$, $J=\left[\begin{array}{cc}0 & -1 \\ 1 & 0\end{array}\right]$ and $g=\lambda_{e}\left[\begin{array}{c}0 \\ \frac{1}{T_{2}}-\frac{1}{u}\end{array}\right]$. The total entropy of the system is given by the sum of the entropies of each compartments $S=S_{1}+S_{2}$. The Poisson bracket $\{S, U\}_{J}$ is then simply the difference of temperatures between the compartments

$$
\{S, U\}_{J}=\frac{\partial S^{\top}}{\partial x} \quad J \frac{\partial U}{\partial x}=\left[\begin{array}{l}
1 \\
1
\end{array}\right]^{\top}\left[\begin{array}{cc}
0 & -1 \\
1 & 0
\end{array}\right]\left[\begin{array}{l}
T_{1} \\
T_{2}
\end{array}\right]=T_{1}-T_{2} .
$$

And one may then identify the expression of the modulating function

$$
R(x, T)=\lambda\left(\frac{1}{T_{2}}-\frac{1}{T_{1}}\right)=\lambda \frac{T_{1}-T_{2}}{T_{1} T 2}=\gamma\left(T_{1}-T_{2}\right),
$$

with $\gamma=\frac{\lambda}{T_{1} T 2}$. Since $\lambda, T_{1}$ and $T_{2}$ are always greater than zero, $\gamma>0$. The vector $g\left(T_{2}, u\right) u$ defines the entropy flow generated by the interaction of subsystem 2 and the external heat source, hence corresponds to the port of the system.

Stability and stabilization We first consider the isolated case (the two systems are isolated from the environment). The availabilty can be used as Lyapunov function and by applying Proposition 1 the system admits an asymptotically stable equilibrium point $\left(S_{1}^{*}, S_{2}^{*}\right)\left(\right.$ with $\left.\frac{\partial U}{\partial x}\left(x^{*}\right)=T^{*}\right)$ if:

$$
\left(T_{2}^{*} T_{1}-T_{1}^{*} T_{2}\right)\left(T_{1}-T_{2}\right) \geq 0
$$

Selecting $T_{1}^{*}=T_{2}^{*}=T^{*}$ we obtain:

$$
T^{*}\left(T_{1}-T_{2}\right)^{2} \geq 0
$$

Equation (19) is only zero if $T_{1}=T_{2}=T^{*}$, which corresponds to the thermodynamic equilibrium of the system. We now consider the controlled system (18). In this case we wish to impose that $T^{*}$ is a desired temperature. Applying Proposition 2 the condition for $x^{*}$ to be an asymptotic stable equilibrium point is

$$
\gamma T^{*}\left(T_{1}-T_{2}\right)^{2}+\left(T^{*}-T_{2}\right)\left(\frac{u-T_{2}}{u T_{2}}\right) \geq 0 .
$$

Hence, the closed-loop system will converge asymptotically to the the desired equilibrium $T^{*}>0$ if the control input is selected such that the previous equation holds. The simplest choice is making the input equal to the desired temperature, i.e., $u=T^{*}$. Notice that the choice $u=T_{2}$ also stabilizes the system, but it is just equivalent to have no control, since compartment 2 and the control compartment are always at same temperature.

\subsection{Example: the CSTR}

The mass balance equations Assume a chemical reaction in a CSTR with the following reversible reaction scheme $\nu_{1} A_{1}+\ldots+\nu_{l} A_{l} \rightleftharpoons \nu_{l+1} A_{l+1}+\ldots+\nu_{m} A_{m}, \quad m>l \geq 1$. The time variation of the species in the reactor are given by (Aris, 1989)

$$
\dot{n}_{i}=F_{e i}-F_{s i}+r_{i} V \quad i=1, \ldots, m
$$

where $n_{i}$ is the number of moles of the species $i$, (and $\mathbf{n}$ the vector $\left.\mathbf{n}=\left(n_{1}, \ldots, n_{m}\right)^{\top}\right), F_{e i}$ and $F_{s i}$ are respectively the inlet and outlet molar flows (and $\mathbf{F}_{e}$ the vector $\mathbf{F}_{e}=$ $\left.\left(F_{e 1}, \ldots, F_{e m}\right)^{\top}\right), r_{i}=\bar{\nu}_{i} r$ where $r(\mathbf{n}, T)$ is the reaction rate which is the difference of the forward reaction rate $r_{f}$ and the backward reaction rate $r_{b}: r=\left(r_{f}-r_{b}\right)$ and depends on the temperature and on the reactant mole number, $\bar{\nu}_{i}$ is the signed stoichiometric coefficient: $\bar{\nu}_{i}=-\nu_{i}$ if it appears on the left hand side of the reaction scheme, $\bar{\nu}_{i}=\nu_{i}$ in the other case. Following the usual assumptions (Aris, 1989; Favache and Dochain, 2009), $V$ 
the volume in the reactor is assumed to be constant as well as the pressure. We shall assume a reaction in gas phase, but the developments may be applied identically to a reactor with a reaction in liquid phase. The assumptions of constant volume and pressure impose a constraint over the total outlet flow $F_{s}\left(\mathbf{n}, T, \mathbf{F}_{e}\right)$ as discussed in (Couenne et al., 2006, 2008), making the outlet flows $F_{s i}=y_{i} F_{s}$ state dependent with $y_{i}=\frac{n_{i}}{\sum_{j=1}^{m} n_{i}}$ being the molar fraction of the species $i$. Furthermore these assumptions guarantee that $A$ is strictly convex.

The energy and entropy balance equations The classical construction of the state space of the ideal mixture in the CSTR is based on Gibbs' relation. Assuming constant volume and pressure of the mixture in the reactor, Gibbs' relation reduces to

$$
d U=\sum_{i=1}^{m} \frac{\partial U}{\partial n_{i}} d n_{i}+\frac{\partial U}{\partial S} d S
$$

where $U$ denotes the internal energy, $S$ the entropy and the conjugated intensive variables are the chemical potential $\frac{\partial U}{\partial n_{i}}=\mu_{i}$ and the temperature $\frac{\partial U}{\partial S}=T$. Gibbs' relation can also be written in the so called entropy formulation

$$
d S=\sum_{i=1}^{m} \frac{\partial S}{\partial n_{i}} d n_{i}+\frac{\partial S}{\partial U} d U
$$

where $\frac{\partial S}{\partial n_{i}}=-\frac{\mu_{i}}{T}$ and $\frac{\partial S}{\partial U}=\frac{1}{T}$ are the intensive thermodynamic variables conjugated to $n_{i}$ and the internal energy $U$. Under the previous assumptions the internal energy of the CSTR is given by

$$
U=\sum_{i=1}^{m} n_{i}\left[c_{p i}\left(T-T_{0}\right)+u_{0 i}\right]
$$

where $c_{p i}, u_{0 i}, T_{0}$ are respectively the heat capacity at constant pressure, reference molar energy and reference temperature. Assuming constant volume and pressure the reference molar enthalpy $h_{0 i}=u_{0 i}$ (Sandler, 2006), and the balance equation of the internal energy is (Couenne et al., 2006; Favache and Dochain, 2009)

$$
\dot{U}=\dot{H}=\sum_{i=1}^{m}\left(F_{e i} h_{e i}-F_{s i} h_{s i}\right)+Q,
$$

where $Q=\lambda\left(T_{e}-T\right)$ is the heat flux from the jacket with $\lambda$ the heat conduction coefficient, $T_{e}$ the temperature of the jacket, $H$ the total enthalpy of the reactor and $h_{e i}, h_{s i}$ respectively the inlet and outlet specific molar enthalpies, which are related with the chemical potentials and the specific molar entropies $s_{i}$ by:

$$
\mu_{i}=h_{i}-T s_{i} .
$$

The entropy function of the CSTR is given by

$$
S=\mathcal{C}_{p} \ln \left(\frac{T}{T_{0}}\right)-R_{g} \sum_{i=1}^{m}\left[n_{i} \ln \left(\frac{n_{i}}{N}\right)\right]+\sum_{i=1}^{m}\left(n_{i} s_{0 i}\right),
$$

where $\mathcal{C}_{p}=\sum_{i=1}^{m} n_{i} c_{p i}, T_{0}, N, s_{0 i}$ and $R_{g}$ are respectively total heat capacity at constant pressure, reference temperature, total number of moles, reference molar entropy and the ideal gas constant. Hence, the entropy balance equation may be deduced from this expression or from Gibbs' relation and is given by

$$
\dot{S}=\sum_{i=1}^{m}\left(F_{e i} s_{e i}-F_{s} s_{i}\right)+\frac{Q}{T_{e}}+\sigma
$$

where $s_{e i}$ and $s_{i}$ are respectively the inlet molar entropy and the molar entropy of species $i$, and $\sigma$ is the irreversible entropy creation due to mass transfer, heat transfer and chemical reactions:

$$
\sigma=\sum_{i=1}^{m} \frac{F_{e i}}{T}\left(h_{e i}-T s_{e i}-\mu_{i}\right)+\frac{Q}{T}-\frac{Q}{T_{e}}-\sum_{i=1}^{m} \mu_{i} \nu_{i} \frac{r}{T} .
$$

IPHS model (Ramirez et al., 2013) The dynamical equation of the CSTR may be expressed as the IPHS

$$
\dot{x}=R J \frac{\partial U}{\partial x}(x)+g_{1}\left(x, \frac{\partial U}{\partial x}\right) u_{1}+g_{2}\left(x, \frac{\partial U}{\partial x}, u_{2}\right)
$$

with state vector $x=\left[n_{1}, \ldots, n_{m}, S\right]^{\top}$, the internal energy $U(x)$ as Hamiltonian function,

$$
J=\left[\begin{array}{cccc}
0 & \ldots & 0 & \bar{\nu}_{1} \\
0 & \ldots & 0 & \vdots \\
0 & \ldots & 0 & \bar{\nu}_{m} \\
-\bar{\nu}_{1} & \ldots & -\bar{\nu}_{m} & 0
\end{array}\right]
$$

a constant skew-symmetric matrix whose elements are the stoichiometric coefficient of the chemical reaction mapping the network structure of the reaction, and

$$
R=\gamma\left(x, \frac{\partial U}{\partial x}\right)\{S, U\}_{J}=\left(\frac{r V}{T \mathcal{A}}\right) \mathcal{A}
$$

with $\gamma=\frac{r V}{T \mathcal{A}}$ and $\{S, U\}_{J}=\mathcal{A}$, where $\mathcal{A}=-\sum_{i=1}^{m} \bar{\nu}_{i} \mu_{i}$ is the chemical affinity of the reaction and corresponds to the thermodynamic driving force of the chemical reaction. The port of the IPHS is given by $g_{1} u_{1}+g_{2}\left(u_{2}\right)$ and is composed by the extended input and output flow vector and the thermal interaction vector defined respectively as

$$
g_{1}=\left[\begin{array}{c}
I-V_{m}^{\dagger} V_{m}^{\top} \\
\left(S_{E}-S_{I} V_{m}^{\dagger} V_{m}^{\top}\right)^{\top}
\end{array}\right], \quad g_{2}=\left[\begin{array}{c}
0 \\
\vdots \\
0 \\
1
\end{array}\right] \frac{h\left(u_{2}-T\right)}{T}
$$

with $V_{m}^{\dagger}=Y\left(V_{m}^{\top} Y\right)^{-1}$ and where $S_{E}=\left[s_{e 1}, \ldots, s_{e m}\right]^{\top}$ and $S_{I}=\left[s_{1}, \ldots, s_{m}\right]^{\top}$ are respectively the inlet molar entropy vector and molar entropy vector, $V_{m}=\left[v_{1}, \ldots, v_{m}\right]^{\top}$ the molar volume vector and $Y=\left[y_{1}, \ldots, y_{m}\right]^{\top}$ the mole fraction vector satisfying $\sum_{i=1}^{m} x_{i}=1$.

Stability and Stabilization The Thermodynamic equilibrium corresponds to a state $x^{*}$ with $\frac{\partial U}{\partial x}\left(x^{*}\right)=$ $\left[\mu_{1}^{*}, \ldots, \mu_{m}^{*}, T^{*}\right]$. Using Proposition 1 we have

$$
\begin{aligned}
& {\left[\begin{array}{c}
\mu_{1}^{*} \\
\vdots \\
\mu_{m}^{*} \\
T^{*}
\end{array}\right]^{\top}\left[\begin{array}{cccc}
0 & \ldots & 0 & \bar{\nu}_{1} \\
0 & \ldots & 0 & \vdots \\
0 & \ldots & 0 & \bar{\nu}_{m} \\
-\bar{\nu}_{1} & \ldots & -\bar{\nu}_{m} & 0
\end{array}\right]\left[\begin{array}{c}
\mu_{1} \\
\vdots \\
\mu_{m} \\
T
\end{array}\right] \mathcal{A} } \\
= & \left(T \sum_{i=1}^{m} \bar{\nu}_{i} \mu_{i}^{*}-T^{*} \sum_{i=1}^{m} \bar{\nu}_{i} \mu_{i}\right) \mathcal{A} \\
= & \left(-T \mathcal{A}^{*}+T^{*} \mathcal{A}\right) \mathcal{A}=-T \mathcal{A}^{*} \mathcal{A}+T^{*} \mathcal{A}^{2} .
\end{aligned}
$$

From Proposition 1 we have that the previous relation should vanish at $\mathcal{A}^{*}$ and be strictly positive for any other state. This imposes the conditions $\mathcal{A}^{*}=0$ and $T^{*}>0$ which corresponds to the thermodynamic equilibrium of the closed system. Let us now consider the controlled IPHS (28). Applying Proposition 2 we have

$$
T^{*} \mathcal{A}^{2}-\left(\frac{\partial U}{\partial x}(x)-\frac{\partial U}{\partial x}\left(x^{*}\right)\right)^{\top}\left(g_{1} u_{1}+g_{2} u_{2}\right) .
$$

where we have used $\mathcal{A}^{*}=0$. Since the first term is positive and vanishes at the desired equilibrium it remains to select 
$u_{1}$ and $u_{2}$ such that the second term is positive and only vanishing at the desired equilibrium. Developing the second term we obtain,

$$
\begin{gathered}
-\left[\left(\mu-\mu^{*}\right)\left(I-V_{m}^{*} V_{m}^{\top}\right)+\frac{T-T^{*}}{T}\left(S_{E}-S_{I} V_{m}^{\dagger} V_{m}^{\top}\right)^{\top}\right] u_{1} \\
-\left(T-T^{*}\right) \frac{\lambda\left(u_{2}-T\right)}{T} .
\end{gathered}
$$

Hence, any physically admissible combination of $u_{1}$ and $u_{2}$ such that (29) fulfils Proposition 2 may be used. In particular, the choice

$$
u_{1}=-\left(\mu-\mu^{*}\right)\left(I-V_{m}^{*} V_{m}^{\top}\right)-\frac{T-T^{*}}{T}\left(S_{E}-S_{I} V_{m}^{\dagger} V_{m}^{\top}\right)^{\top},
$$$$
u_{2}=T^{*} \text {. }
$$

asymptotically stabilizes the closed-loop CSTR at the desired equilibrium $x^{*}=\left[\mu_{1}^{*}, \ldots, \mu_{m}^{*}, T^{*}\right]$.

\section{CONCLUSION}

Irreversible port Hamiltonian systems (IPHS) have been used to derive a class of passivity based controllers for irreversible thermodynamic systems. The energy based availability function has been defined and specialized to IPHS. Unlike previous work, where the total entropy usually corresponds to the generating function, the generating function of the energy based availability is the internal energy of a thermodynamic system. Since the internal energy corresponds to the Hamiltonian of IPHS, the structure of IPHS permits to elegantly derive stability conditions for open and closed thermodynamic systems. It turns out that for this class of systems the energy based availability function defines naturally a Lyapunov function. In order to guarantee the convexity of the availability function a constraint on the mass or the volume has to be imposed. This constraint (constant mass or volume) is a standard assumption in the control of chemical reactors, hence it is not restrictive. The proposed approach has then been used for the passivity based control design for irreversible thermodynamic systems in an analogous manner as passivity based control techniques in mechanical systems. The results have been illustrated on two classical thermodynamic models: The heat exchanger and the continuous stirred tank reactor.

\section{REFERENCES}

Alonso, A.A. and Ydstie, B.E. (1996). Process systems, passivity and the second law of thermodynamics. Computers and Chemical Engineering, 20, 1119-1124.

Alonso, A.A. and Ydstie, B.E. (2001). Stabilization of distributed systems using irreversible thermodynamics. Automatica, 37, 17391755.

Aris, R. (1989). Elementary chemical reactor analysis. Chemical Engineering. Butterworths, Stoneham, USA.

Brockett, R. (1977). Control theory and analytical mechanics. In C. Martin and R. Hermann (eds.), Geometric Control Theory, 1-46. Math Sci Press, Brookline, USA.

Callen, H. (1985). Thermodynamics and an introduction to thermostatistics. Wiley, New-York.

Couenne, F., Jallut, C., Maschke, B., Breedveld, P., and Tayakout, M. (2006). Bond graph modelling for chemical reactors. Mathematical and Computer Modelling of Dynamical Systems, 12(2-3), 159-174.

Couenne, F., Jallut, C., Maschke, B., Tayakout, M., and Breedveld, P. (2008). Structured modeling for processes: A thermodynamical network theory. Computers \& Chemical Engineering, 32(6), 1120 1134 .

Dörfler, F., Johnsen, J., and Allgöwer, F. (2009). An introduction to interconnection and damping assignment passivity-based control in process engineering. Journal of Process Control, 19, 1413-1426.
Duindam, V., Macchelli, A., Stramigioli, S., and Bruyninckx, H. (eds.) (2009). Modeling and Control of Complex Physical Systems - The Port-Hamiltonian Approach. Springer-Verlag, Berlin, Germany.

Eberard, D., Maschke, B.M., and van der Schaft, A.J. (2007). An extension of Hamiltonian systems to the thermodynamic phase space: Towards a geometry of nonreversible processes. Reports on Mathematical Physics, 60, 175-198.

Favache, A. and Dochain, D. (2009). Thermodynamics and chemical systems stability: The CSTR case study revisited. Journal of Process Control, 19, 371-379.

Favache, A., Dochain, D., and Maschke, B. (2010). An entropy-based formulation of irreversible processes based on contact structures. Chemical Engineering Science, 65, 5204-5216.

Hangos, K.M., Bokor, J., and Szederkényi, G. (2001). Hamiltonian view on process systems. AIChE Journal, 47, 1819-1831.

Hoang, H., Couenne, F., Jallut, C., and Le Gorrec, Y. (2011). The port Hamiltonian approach to modelling and control of continuous stirred tank reactors. Journal of Process Control, 21(10), 1449-1458.

Hoang, H., Couenne, F., Jallut, C., and Le Gorrec, Y. (2012). Lyapunovbased control of non isothermal continuous stirred tank reactors using irreversible thermodynamics. Journal of Process Control, 22(2), 412422 .

Jillson, K.R. and Ydstie, B.E. (2007). Process networks with decentralized inventory and flow control. Journal of Process Control, 17(5), $399-413$

Maschke, B. and van der Schaft, A. (1992). Port controlled Hamiltonian systems: modeling origins and system theoretic properties. In Proceedings of the 3rd IFAC Symposium on Nonlinear Control Systems, NOLCOS'92, 282-288. Bordeaux, France.

Maschke, B., van der Schaft, A., and Breedveld, P. (1992). An intrinsic Hamiltonian formulation of network dynamics: Non-standard Poisson structures and gyrators. Journal of the Franklin Institute, 329(5), 923-966.

Ortega, R., Loria, A., Nicklasson, P., and Sira-Ramirez, H. (1998). Passivity-based control of Euler-Lagrange Systems. Communications and Control Series. Springer, Berlin.

Ortega, R., van der Schaft, A., Maschke, B., and Escobar, G. (2002). Interconnection and damping assignment passivity based control of port-controlled Hamiltonian systems. Automatica, 38, 585-596.

Otero-Muras, I., Szederkényi, G., Alonso, A.A., and Hangos, K.M. (2008). Local dissipative Hamiltonian description of reversible reaction networks. Systems \& Control Letters, 57, 554-560.

Ramirez, H. (2012). Control of irreversible thermodynamic processes using port-Hamiltonian systems defined on pseudo-Poisson and contact structures. Ph.D. thesis, Université Claude Bernard Lyon

Ramirez, H., Maschke, B., and Sbarbaro, D. (2012). Irreversible portHamiltonian systems. In Proceedings of the 4th IFAC workshop on Lagrangian and Hamiltonian methods for nonlinear control ( $L H M N L C)$. Bertinoro, Italy.

Ramirez, H., Sbarbaro, D., and Ortega, R. (2009). On the control of nonlinear processes: An IDA-PBC approach. Journal of Process Control, 19, 405-414.

Ramirez, H., Maschke, B., and Sbarbaro, D. (2013). Irreversible portHamiltonian systems: A general formulation of irreversible processes with application to the CSTR. Chemical Engineering Science, 89(0), $223-234$

Sandler, S. (2006). Chemical, Biochemical, and Engineering Thermodynamics. John Wiley \& Sons, Hoboken, USA, fourth edition.

van der Schaft, A.J. (2000). L2-Gain and Passivity Techniques in Nonlinear Control.

van der Schaft, A. (1986). On feedback control of Hamiltonian systems. In C.I. Byrnes and A. Lindquist (eds.), Theory and Applications of Nonlinear Control Systems, 273-290. Elsevier North-Holland, New York, USA.

van der Schaft, A. and Maschke, B. (1994). On the Hamiltonian formulation of non-holonomic mechanical systems. Reports on Mathematical Physics, 34(2), 225-233.

Willems, J. (1972). Dissipative dynamical systems part I: General theory. Archive for Rational Mechanics and Analysis, 45, 321-351.

Ydstie, B.E. (2002). Passivity based control via the second law. Computers and Chemical Engineering, 26, 1037-1048.

Ydstie, B.E. and Alonso, A.A. (1997). Process systems and passivity via the clausius-planck inequality. Systems \&s Control Letters, 30(5), 253 -264 . 